\title{
Invariant Distributionally Scrambled Manifolds for an Annihilation Operator
}

\author{
Xinxing Wu \\ School of Mathematics, University of Electronic Science and Technology of China, Chengdu, Sichuan 611731, China \\ Correspondence should be addressed to Xinxing Wu; wuxinxing5201314@163.com
}

Received 20 January 2014; Accepted 15 May 2014; Published 27 May 2014

Academic Editor: Alfredo Peris

Copyright (C) 2014 Xinxing Wu. This is an open access article distributed under the Creative Commons Attribution License, which permits unrestricted use, distribution, and reproduction in any medium, provided the original work is properly cited.

This note proves that the annihilation operator of a quantum harmonic oscillator admits an invariant distributionally $\varepsilon$-scrambled linear manifold for any $0<\varepsilon<2$. This is a positive answer to Question 1 by Wu and Chen (2013).

A dynamical system is a pair $(X, f)$, where $X$ is a complete metric space without isolated points and the map $f: X \rightarrow X$ is continuous. Throughout this paper, let $\mathbb{N}=\{1,2,3, \ldots\}$ and $\mathbb{Z}^{+}=\{0,1,2, \ldots\}$.

Sharkovskii's amazing discovery [1], as well as Li and Yorke's famous work which introduced the concept of "chaos" known as the Li-Yorke chaos today in a mathematically rigorous way [2], has activated sustained interest and provoked the rapid advancement of discrete chaos theory in the last decades. Since then, several other rigorous definitions of chaos have been proposed. Each of their definitions tries to describe one kind of unpredictability in the evolution of the system dynamics. This was also the original idea of $\mathrm{Li}$ and Yorke. In Li and Yorke's study [2], they suggested considering "divergent pairs" $(x, y)$, which are proximal but not asymptotic, in the sense that

$$
\begin{aligned}
& \liminf _{n \rightarrow \infty} d\left(f^{n}(x), f^{n}(y)\right)=0, \\
& \limsup _{n \rightarrow \infty} d\left(f^{n}(x), f^{n}(y)\right)>0,
\end{aligned}
$$

where $f^{n}$ denotes the $n$th iteration of $f$.

A generalization of the concept of Li-Yorke chaos is distributional chaos, introduced by Schweizer and Smítal [3] in 1994.
Let $(X, f)$ be a dynamical system. For any pair of points $x, y \in X$ and any $n \in \mathbb{N}$, let

$$
F(x, y, t, n)=\left|\left\{j \in \mathbb{N}: d\left(f^{j}(x), f^{j}(y)\right)<t, 1 \leq j \leq n\right\}\right|,
$$

where $|A|$ denotes the cardinality of set $A$. Define lower and upper distributional functions $\mathbb{R} \rightarrow[0,1]$ generated by $f, x$, and $y$, as

$$
\begin{aligned}
& F_{x, y}(t, f)=\liminf _{n \rightarrow \infty} \frac{1}{n} F(x, y, t, n), \\
& F_{x, y}^{*}(t, f)=\limsup _{n \rightarrow \infty} \frac{1}{n} F(x, y, t, n),
\end{aligned}
$$

respectively. A dynamical system $(X, f)$ is said to be distributionally $\varepsilon$-chaotic for a given $\varepsilon>0$ if there exists an uncountable subset $D \subset X$ such that for any pair of distinct points $x, y \in D$, one has $F_{x, y}^{*}(t, f)=1$ for all $t>0$ and $F_{x, y}(\varepsilon, f)=0$. The set $D$ is a distributionally $\varepsilon$-scrambled set and the pair $(x, y)$ a distributionally $\varepsilon$-chaotic pair. If $(X, f)$ is distributionally $\varepsilon$-chaotic for any given $0<\varepsilon<\operatorname{diam} X$, then $(X, f)$ is said to exhibit maximal distributional chaos.

The quantum harmonic oscillator is the quantummechanical analog of the classical harmonic oscillator. It is one of the most important models in quantum mechanics $[4,5]$, because an arbitrary potential can be approximated by 
a harmonic potential at the vicinity of a stable equilibrium point. Transmutation of the quantum harmonic oscillator may be described by the (time-dependent) Schrödinger equation as

$$
-\frac{\hbar^{2}}{2 m} \frac{\partial^{2} \psi}{\partial x^{2}}+\frac{m \omega^{2}}{2} x^{2} \psi=i \hbar \frac{\partial \psi}{\partial t}
$$

with a wave function $\psi(x, t)$, displacement $x$, mass $m$, frequency $\omega$, and Planck constant $\hbar$. The nondimensionalized steady states in terms of eigenfunctions in the separable Hilbert space $\mathscr{H}=L^{2}(-\infty,+\infty)$ form an orthonormal basis:

$$
\psi_{n}(x)=\frac{e^{-x^{2} / 2} H_{n}(x)}{\sqrt{\sqrt{\pi} 2^{n} n !}}, \quad n=0,1, \ldots
$$

where $H_{n}(x)=(-1)^{n} e^{x^{2}}\left(\mathrm{~d}^{n} / \mathrm{d} x^{n}\right) e^{-x^{2}}$ is the $n$th Hermite polynomial. The natural phase space for the quantum harmonic oscillator is the Schwartz class, also called Schwartz space $\Phi$ of rapidly decreasing functions in $\mathscr{H}$, defined as

$$
\Phi=\left\{\phi \in \mathscr{H}: \phi=\sum_{n=0}^{\infty} c_{n} \psi_{n}, \sum_{n=0}^{\infty}\left|c_{n}\right|^{2}(n+1)^{r}<+\infty, \forall r>0\right\} .
$$

Here, $\Phi$ is an infinite-dimensional Fréchet space with topology defined by the system of seminorms $p_{r}(\cdot)$ of the form

$$
p_{r}(\phi)=p_{r}\left(\sum_{n=0}^{\infty} c_{n} \psi_{n}\right)=\left(\sum_{n=0}^{\infty}\left|c_{n}\right|^{2}(n+1)^{r}\right)^{1 / 2}
$$

This topology on $\Phi$ can be equivalently introduced by the metric

$$
\rho(\phi, \psi)=\sum_{m=0}^{\infty} \frac{1}{2^{m}} \frac{p_{m}(\phi-\psi)}{1+p_{m}(\phi-\psi)} .
$$

It follows directly from (9) that $\operatorname{diam} \Phi=2$. For any $\phi=$ $\sum_{n=0}^{\infty} c_{n} \psi_{n} \in \Phi$ and any $i \in \mathbb{Z}^{+}$, denote $\phi_{i}=c_{i}$. The quantum harmonic oscillator may be equivalently described in terms of the annihilation operator $\widehat{a}=(1 / \sqrt{2})(x+(\mathrm{d} / \mathrm{d} x))$ and its adjoint $a^{\dagger}=(1 / \sqrt{2})(x-(\mathrm{d} / \mathrm{d} x))$. According to the basic properties of Hermite polynomials, one has $\widehat{a}: \Phi \rightarrow \Phi$ given by

$$
\widehat{a}\left(\psi_{n}\right)=\frac{1}{\sqrt{2}}\left(x+\frac{\mathrm{d}}{\mathrm{d} x}\right) \psi_{n}=\sqrt{n} \psi_{n-1}
$$

Meanwhile, it is not difficult to check that for any $i \in \mathbb{N}$ and any $\phi=\sum_{n=0}^{\infty} c_{n} \psi_{n} \in \Phi$,

$$
\widehat{a}^{i}(\phi)=\sum_{n=i}^{\infty} \sqrt{A_{n}^{i}} \cdot c_{n} \cdot \psi_{n-i}=\sum_{n=0}^{\infty} \sqrt{A_{n+i}^{i}} \cdot c_{n+i} \cdot \psi_{n},
$$

where $A_{n}^{i}=n \cdot(n-1) \cdots(n-i+1)$. The $\widehat{a}$ acts as a kind of backward shift on the space $\Phi$. In fact, it is a special weighted backward shift on the Fréchet space (see [6-8] for the recent results on this topic).
Applying a result of Godefroy and Shapiro [9], Gulisashvili and MacCluer [10] proved that the annihilation operator $\widehat{a}$ is Devaney chaotic. Then, Duan et al. [11] obtained that $\widehat{a}$ is also Li-Yorke chaotic. However, it follows directly from [12, Theorem 4.1] that this holds trivially. Oprocha [13] showed that $\widehat{a}$ is distributionally $\varepsilon$-chaotic with $\varepsilon=1 / 16$. Recently, in [14] it was further shown that $\hat{a}$ exhibits distributional $\varepsilon$ chaos for any $0<\varepsilon<2$ and that the principal measure of $\widehat{a}$ is 1 . Moreover, Wu and Chen [15] proved that $\widehat{a}$ admits an invariant distributionally $\varepsilon$-scrambled set for any $0<\varepsilon<2$ and posed the following question.

Question. Is there an invariant manifold $D$ of $\Phi$ such that $D$ is a distributionally $\varepsilon$-scrambled set for any $0<\varepsilon<2$ ?

This paper gives a positive answer to the question above; see the following theorem.

Theorem 1. There exists an invariant manifold $E \subset \Phi$ such that $E$ is a distributionally $\varepsilon$-scrambled set under $\widehat{a}$ for any $0<$ $\varepsilon<2=\operatorname{diam} \Phi$.

Proof. Let $L_{1}=\mathscr{L}_{1}=2, L_{n}=2^{L_{1}+\cdots+L_{n-1}}$, and $\mathscr{L}_{n}=\sum_{j=1}^{n} L_{j}$ for $n>1$. Arrange all odd prime numbers by the natural order " $<$ " and denote them by $\mathrm{P}_{1}, \mathrm{P}_{2}, \ldots$ For any $n, m \in \mathbb{N}$, set

$$
\mathscr{A}_{n, m}=\left\{j \in \mathbb{Z}^{+}: \mathscr{L}_{\mathrm{P}_{n}^{m}} \leq j<\mathscr{L}_{\mathrm{P}_{n}^{m}+1}, j-\mathscr{L}_{\mathrm{P}_{n}^{m}} \equiv 0(\bmod )\right\} .
$$

Take a point $\xi=\sum_{j=0}^{\infty} \xi_{j} \psi_{j}$ such that

$$
\xi_{j}= \begin{cases}\sqrt{\frac{\mathrm{P}_{n}^{m}}{j !}}, & j \in \mathscr{A}_{n, m}, n, m \in \mathbb{N} \\ 0, & \text { otherwise. }\end{cases}
$$

Since $j \geq \mathscr{L}_{\mathrm{P}_{n}^{m}} \geq \mathrm{P}_{n}^{m}$ if $j \in \mathscr{A}_{n, m}$, we have that, for any $j \in \mathbb{N}$,

$$
\left|\xi_{j}\right| \leq \frac{1}{\sqrt{(j-1) !}}
$$

This implies that, for any $r \geq 0$,

$$
\sum_{j=0}^{\infty}\left|\xi_{j}\right|^{2}(j+1)^{r} \leq \sum_{j=1}^{\infty} \frac{1}{(j-1) !}(j+1)^{r}<+\infty .
$$

Hence $\xi \in \Phi$.

Take $E:=\operatorname{span}\left\{\xi^{(n)}: n \in \mathbb{Z}^{+}\right\}$, where $\xi^{(n)}=\widehat{a}^{n}(\xi)$. Clearly, $E$ is an invariant linear manifold under $\widehat{a}$. Given two fixed points $\phi, \psi \in E$ with $\phi \neq \psi$, according to the construction of $E$, there exist $\alpha_{0}, \beta_{0}, \ldots, \alpha_{N}, \beta_{N} \in \mathbb{K}$, such that $\phi=\alpha_{0} \xi^{(0)}+$ $\cdots+\alpha_{N} \xi^{(N)}$ and $\psi=\beta_{0} \xi^{(0)}+\cdots+\beta_{N} \xi^{(N)}$.

Now, we assert that $(\phi, \psi)$ is a distributionally $\varepsilon$-chaotic pair for any $0<\varepsilon<2$.

First, observe that for any $\mathscr{L}_{2 n} \leq j<\mathscr{L}_{2 n+1}, \xi_{j}=0$ and $\hat{a}^{j}(\xi)=\sum_{n=0}^{\infty} \sqrt{A_{n+j}^{j}} \cdot \xi_{n+j} \cdot \psi_{n}$. Combining this with (14), it 
follows that for any fixed $m \in \mathbb{Z}^{+}$and any $\mathscr{L}_{2 n} \leq j \leq \mathscr{L}_{2 n}+$ $\left(L_{2 n+1} / 2\right)$,

$$
\begin{aligned}
p_{m}\left(\widehat{a}^{j}(\xi)\right) & =\left(\sum_{k=0}^{\infty}\left|\sqrt{A_{k+j}^{j}} \cdot \xi_{k+j}\right|^{2}(k+1)^{m}\right)^{1 / 2} \\
& \leq\left(\sum_{k=\mathscr{L}_{2 n+1}-(j+1)}^{\infty}\left|\sqrt{A_{k+j}^{j}} \cdot \xi_{k+j}\right|^{2}(k+1)^{m}\right)^{1 / 2} \\
& \leq\left(\sum_{k=\left(L_{2 n+1} / 2\right)-1}^{\infty} \frac{k+j}{k !}(k+1)^{m}\right)^{1 / 2}
\end{aligned}
$$

as $\mathscr{L}_{2 n+1}-(j+1) \geq \mathscr{L}_{2 n+1}-\left(\mathscr{L}_{2 n}+\left(L_{2 n+1} / 2\right)+1\right) \geq\left(L_{2 n+1} / 2\right)-$ 1. Meanwhile, it is easy to see that, for any $\mathscr{L}_{2 n} \leq j \leq \mathscr{L}_{2 n}+$ $\left(L_{2 n+1} / 2\right)$,

$$
\begin{aligned}
& \sum_{k=\left(L_{2 n+1} / 2\right)-1}^{\infty} \frac{k+j}{k !}(k+1)^{m} \\
& \quad \leq \sum_{k=\left(L_{2 n+1} / 2\right)-1}^{\infty} \frac{k+\mathscr{L}_{2 n}+\left(L_{2 n+1} / 2\right)}{k !}(k+1)^{m} \\
& =\sum_{k=\left(L_{2 n+1} / 2\right)-1}^{\infty} \frac{k+\log _{2} L_{2 n+1}+\left(L_{2 n+1} / 2\right)}{k !}(k+1)^{m} \longrightarrow 0
\end{aligned}
$$

For any fixed $t>0$, one can choose a $K_{1} \in \mathbb{N}$ such that $\sum_{n=K_{1}+1}^{\infty}\left(1 / 2^{n}\right)<(t / 2(N+1))$. It is clear that, for any $j, m \in$ $\mathbb{Z}^{+}$

$$
p_{m}\left(\widehat{a}^{j}(\phi)-\widehat{a}^{j}(\psi)\right) \leq \sum_{k=0}^{N}\left|\alpha_{k}-\beta_{k}\right| \cdot p_{m}\left(\widehat{a}^{j+k}(\xi)\right) .
$$

This, together with (16) and (17), leads to that there exists a $K_{2} \in \mathbb{N}$ such that for any $n \geq K_{2}$ and any $\mathscr{L}_{2 n} \leq j \leq \mathscr{L}_{2 n}+$ $\left(L_{2 n+1} / 2\right)-N$,

$$
\begin{aligned}
\max & \left\{\left|\alpha_{k}-\beta_{k}\right| \cdot p_{m}\left(\widehat{a}^{j+k}(\xi)\right): 0 \leq k \leq N, 0 \leq m \leq K_{1}\right\} \\
& <\frac{t}{4(N+1)} .
\end{aligned}
$$

This implies that, for any $\mathscr{L}_{2 n} \leq j \leq \mathscr{L}_{2 n}+\left(L_{2 n+1} / 2\right)-$ $N\left(n \geq K_{2}\right)$,

$$
\begin{aligned}
& \rho\left(\widehat{a}^{j}(\phi), \widehat{a}^{j}(\psi)\right) \\
& \quad \leq \sum_{k=0}^{N} \rho\left(\widehat{a}^{j+k}\left(\alpha_{k} \xi\right), \widehat{a}^{j+k}\left(\beta_{k} \xi\right)\right) \\
& \quad \leq \sum_{k=0}^{N}\left(\sum_{m=0}^{K_{1}} \frac{1}{2^{m}} \frac{\left|\alpha_{k}-\beta_{k}\right| \cdot p_{m}\left(\widehat{a}^{j+k}(\xi)\right)}{1+\left|\alpha_{k}-\beta_{k}\right| \cdot p_{m}\left(\widehat{a}^{j+k}(\xi)\right)}+\sum_{m=K_{1}+1}^{\infty} \frac{1}{2^{m}}\right)
\end{aligned}
$$

$$
\begin{aligned}
& <\sum_{k=0}^{N}\left(\sum_{m=0}^{K_{1}} \frac{1}{2^{m}} \frac{(t / 4(N+1))}{1+(t / 4(N+1))}+\frac{t}{2(N+1)}\right) \\
& <\sum_{k=0}^{N} \frac{t}{N+1}=t .
\end{aligned}
$$

Consequently,

$$
\begin{aligned}
& F_{\phi, \psi}^{*}(t, \widehat{a}) \\
& \quad=\limsup _{n \rightarrow \infty} \frac{1}{n}\left|\left\{j: \rho\left(\widehat{a}^{j}(\phi), \widehat{a}^{j}(\psi)\right)<t, 1 \leq j \leq n\right\}\right| \\
& \geq \limsup _{n \rightarrow \infty} \frac{1}{\mathscr{L}_{2 n}+\left(L_{2 n+1} / 2\right)-N} \\
& \quad \times\left|\left\{j: \rho\left(\widehat{a}^{j}(\psi), \widehat{a}^{j}(\phi)\right)<t, 1 \leq j \leq \mathscr{L}_{2 n}+\frac{L_{2 n+1}}{2}-N\right\}\right| \\
& \geq \limsup _{n \rightarrow \infty} \frac{\left(L_{2 n+1} / 2\right)-N}{\mathscr{L}_{2 n}+\left(L_{2 n+1} / 2\right)-N} \\
& =\limsup _{n \rightarrow \infty} \frac{2^{\mathscr{L}_{2 n}-1}-N}{\mathscr{L}_{2 n}+2^{\mathscr{L}_{2 n}-1}-N}=1 .
\end{aligned}
$$

Second, since $\phi \neq \psi$, there exists $0 \leq \ell \leq N$ such that $\alpha_{\ell} \neq \beta_{\ell}$. It follows from (11) that, for any $j \in \mathbb{Z}^{+}$,

$$
\begin{aligned}
\widehat{a}^{j}(\phi)-\widehat{a}^{j}(\psi) \\
=\sum_{k=0}^{N}\left(\alpha_{k}-\beta_{k}\right) \cdot \widehat{a}^{j+k}(\xi) \\
=\sum_{n=0}^{\infty}\left(\sum_{k=0}^{N}\left(\alpha_{k}-\beta_{k}\right) \cdot \sqrt{A_{n+j+k}^{j+k}} \cdot \xi_{n+j+k} \cdot \psi_{n}\right) .
\end{aligned}
$$

For any fixed $\mathscr{L}_{\mathrm{P}_{n}^{N+1}} \leq j \leq \mathscr{L}_{\mathrm{P}_{n}^{N+1}}+\left(L_{\mathrm{P}_{n}^{N+1}+1} / 2\right)(n \geq N)$, there exists $0 \leq i_{0} \leq N$ such that $j+\ell+i_{0} \in \mathscr{A}_{n, N+1}$. According to the choice of $\xi$, it follows that for all $i \in[0, N] \backslash\left\{i_{0}\right\}, \xi_{j+\ell+i}=0$. This implies that

$$
\begin{aligned}
& \left(\widehat{a}^{j}(\phi)-\widehat{a}^{j}(\psi)\right)_{i_{0}} \\
& \quad=\left(\alpha_{\ell}-\beta_{\ell}\right) \cdot \sqrt{A_{i_{0}+j+\ell}^{j+\ell}} \cdot \sqrt{\frac{\mathrm{P}_{n}^{N+1}}{\left(i_{0}+j+\ell\right) !}} \\
& \quad=\left(\alpha_{\ell}-\beta_{\ell}\right) \cdot \sqrt{\frac{\mathrm{P}_{n}^{N+1}}{i_{0} !}} .
\end{aligned}
$$


Then, for any $m \in \mathbb{Z}^{+}$,

$$
\begin{aligned}
p_{m} & \left(\widehat{a}^{j}(\phi)-\widehat{a}^{j}(\psi)\right) \\
& \geq p_{0}\left(\widehat{a}^{j}(\phi)-\widehat{a}^{j}(\psi)\right) \\
& \geq\left|\alpha_{\ell}-\beta_{\ell}\right| \cdot \sqrt{\frac{\mathrm{P}_{n}^{N+1}}{i_{0} !}} \\
& \geq\left|\alpha_{\ell}-\beta_{\ell}\right| \cdot \sqrt{\frac{\mathrm{P}_{n}^{N+1}}{N !}} .
\end{aligned}
$$

Combining this with the fact that the function $t \in[0,+\infty) \mapsto$ $(t /(1+t)) \in \mathbb{R}$ is increasing, it follows that, for any $\mathscr{L}_{\mathrm{P}_{n}^{N+1}} \leq$ $j \leq \mathscr{L}_{\mathrm{P}_{n}^{N+1}}+\left(L_{\mathrm{P}_{n}^{N+1}+1} / 2\right)$,

$$
\begin{aligned}
& \rho\left(\widehat{a}^{j}(\phi), \hat{a}^{j}(\psi)\right) \\
& \quad=\sum_{m=0}^{\infty} \frac{1}{2^{m}} \frac{p_{m}\left(\widehat{a}^{j}(\phi)-\widehat{a}^{j}(\psi)\right)}{1+p_{m}\left(\hat{a}^{j}(\phi)-\widehat{a}^{j}(\psi)\right)} \\
& \quad \geq \sum_{m=0}^{\infty} \frac{1}{2^{m}} \cdot \frac{\left|\alpha_{\ell}-\beta_{\ell}\right| \cdot \sqrt{\left(\mathrm{P}_{n}^{N+1} / N !\right)}}{1+\left|\alpha_{\ell}-\beta_{\ell}\right| \cdot \sqrt{\left(\mathrm{P}_{n}^{N+1} / N !\right)}} \\
& \quad=\frac{2\left|\alpha_{\ell}-\beta_{\ell}\right| \cdot \sqrt{\left(\mathrm{P}_{n}^{N+1} / N !\right)}}{1+\left|\alpha_{\ell}-\beta_{\ell}\right| \cdot \sqrt{\left(\mathrm{P}_{n}^{N+1} / N !\right)}} \longrightarrow 2 \quad(n \longrightarrow+\infty) .
\end{aligned}
$$

Hence, for any $0<\varepsilon<2$,

$$
\begin{aligned}
& F_{\phi, \psi}(\varepsilon, \widehat{a}) \\
& =\liminf _{n \rightarrow \infty} \frac{1}{n}\left|\left\{j: \rho\left(\widehat{a}^{j}(\phi), \widehat{a}^{j}(\psi)\right)<\varepsilon, 1 \leq j \leq n\right\}\right| \\
& \leq \liminf _{n \rightarrow \infty} \frac{1}{\mathscr{L}_{\mathrm{P}_{n}^{N+1}}+\left(L_{\mathrm{P}_{n}^{N+1}+1} / 2\right)} \\
& \quad \times\left|\left\{j: \rho\left(\widehat{a}^{j}(\phi), \widehat{a}^{j}(\psi)\right)<\varepsilon, 1 \leq j \leq \mathscr{L}_{\mathrm{P}_{n}^{N+1}}+\frac{L_{\mathrm{P}_{n}^{N+1}+1}}{2}\right\}\right| \\
& \leq \liminf _{n \rightarrow \infty} \frac{\mathscr{L}_{\mathrm{P}_{n}^{N+1}}}{\mathscr{L}_{\mathrm{P}_{n}^{N+1}}+\left(L_{\mathrm{P}_{n}^{N+1}+1} / 2\right)} \\
& =\liminf _{n \rightarrow \infty} \frac{\mathscr{L}_{\mathrm{P}_{n}^{N+1}}}{\mathscr{L}_{\mathrm{P}_{n}^{N+1}}+2^{\mathscr{L}_{\mathrm{P}_{n}^{N+1}}-1}}=0 .
\end{aligned}
$$

Summing up the above discussions, since both $\phi$ and $\psi$ are arbitrary, it follows that $E$ is an invariant distributionally $\varepsilon$-scrambled linear manifold for any $0<\varepsilon<2$.

\section{Conflict of Interests}

The authors declare that there is no conflict of interests regarding the publication of this paper.

\section{Acknowledgments}

This paper was completed during the author's visit to the City University of Hong Kong. This paper was supported by YBXSZC20131046, the Scientific Research Fund of Sichuan Provincial Education Department (no. 14ZB0007).

\section{References}

[1] O. M. Sharkovskii, "Co-existence of cycles of a continuous mapping of the line into itself," Ukrainskiu Matematicheskiu Zhurnal, vol. 16, pp. 61-71, 1964.

[2] T. Y. Li and J. A. Yorke, "Period three implies chaos," The American Mathematical Monthly, vol. 82, no. 10, pp. 985-992, 1975.

[3] B. Schweizer and J. Smítal, "Measures of chaos and a spectral decomposition of dynamical systems on the interval," Transactions of the American Mathematical Society, vol. 344, no. 2, pp. 737-754, 1994.

[4] R. L. Liboff, Introductory Quantum Mechanics, Addison-Wesley, Reading, Mass, USA, 3rd edition, 1998.

[5] J. Singh, Quantum Mechanics: Fundamentals and Applications to Technology, Wiley, New York, NY, USA, 1997.

[6] T. Bermúdez, A. Bonilla, F. Martínez-Giménez, and A. Peris, "Li-Yorke and distributionally chaotic operators," Journal of Mathematical Analysis and Applications, vol. 373, no. 1, pp. 8393, 2011.

[7] N. C. Bernardes Jr., A. Bonilla, V. Müller, and A. Peris, "Distributional chaos for linear operators," Journal of Functional Analysis, vol. 265, no. 9, pp. 2143-2163, 2013.

[8] F. Martínez-Giménez, P. Oprocha, and A. Peris, "Distributional chaos for backward shifts," Journal of Mathematical Analysis and Applications, vol. 351, no. 2, pp. 607-615, 2009.

[9] G. Godefroy and J. H. Shapiro, "Operators with dense, invariant, cyclic vector manifolds," Journal of Functional Analysis, vol. 98, no. 2, pp. 229-269, 1991.

[10] A. Gulisashvili and C. R. MacCluer, "Linear chaos in the unforced quantum harmonic oscillator," Journal of Dynamic Systems, Measurement, and Control, vol. 118, pp. 337-338, 1996.

[11] J. Duan, X.-C. Fu, P.-D. Liu, and A. Manning, "A linear chaotic quantum harmonic oscillator," Applied Mathematics Letters, vol. 12, no. 1, pp. 15-19, 1999.

[12] W. Huang and X. Ye, "Devaney's chaos or 2-scattering implies Li-Yorke's chaos," Topology and Its Applications, vol. 117, no. 3, pp. 259-272, 2002.

[13] P. Oprocha, "A quantum harmonic oscillator and strong chaos," Journal of Physics A, vol. 39, no. 47, pp. 14559-14565, 2006.

[14] X. Wu and P. Zhu, "The principal measure of a quantum harmonic oscillator," Journal of Physics A, vol. 44, no. 50, Article ID 505101, 2011.

[15] $\mathrm{X}$. Wu and G. Chen, "On the invariance of maximal distributional chaos under an annihilation operator," Applied Mathematics Letters, vol. 16, pp. 1134-1140, 2013. 


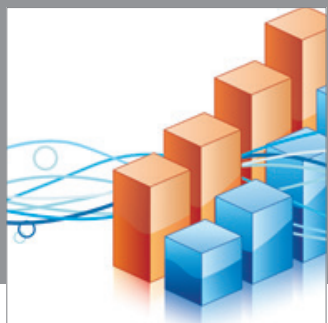

Advances in

Operations Research

mansans

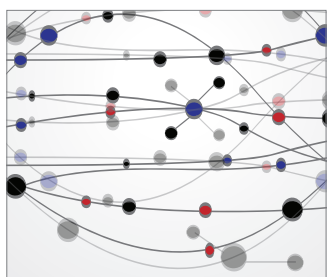

The Scientific World Journal
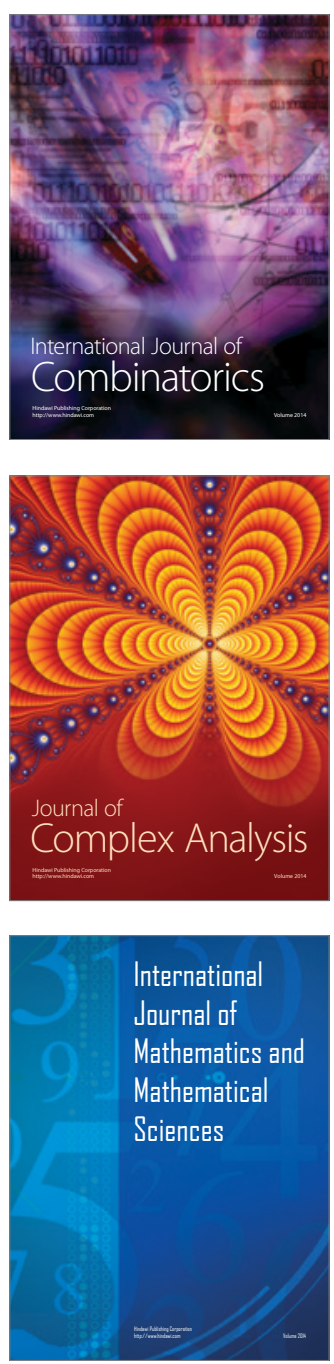
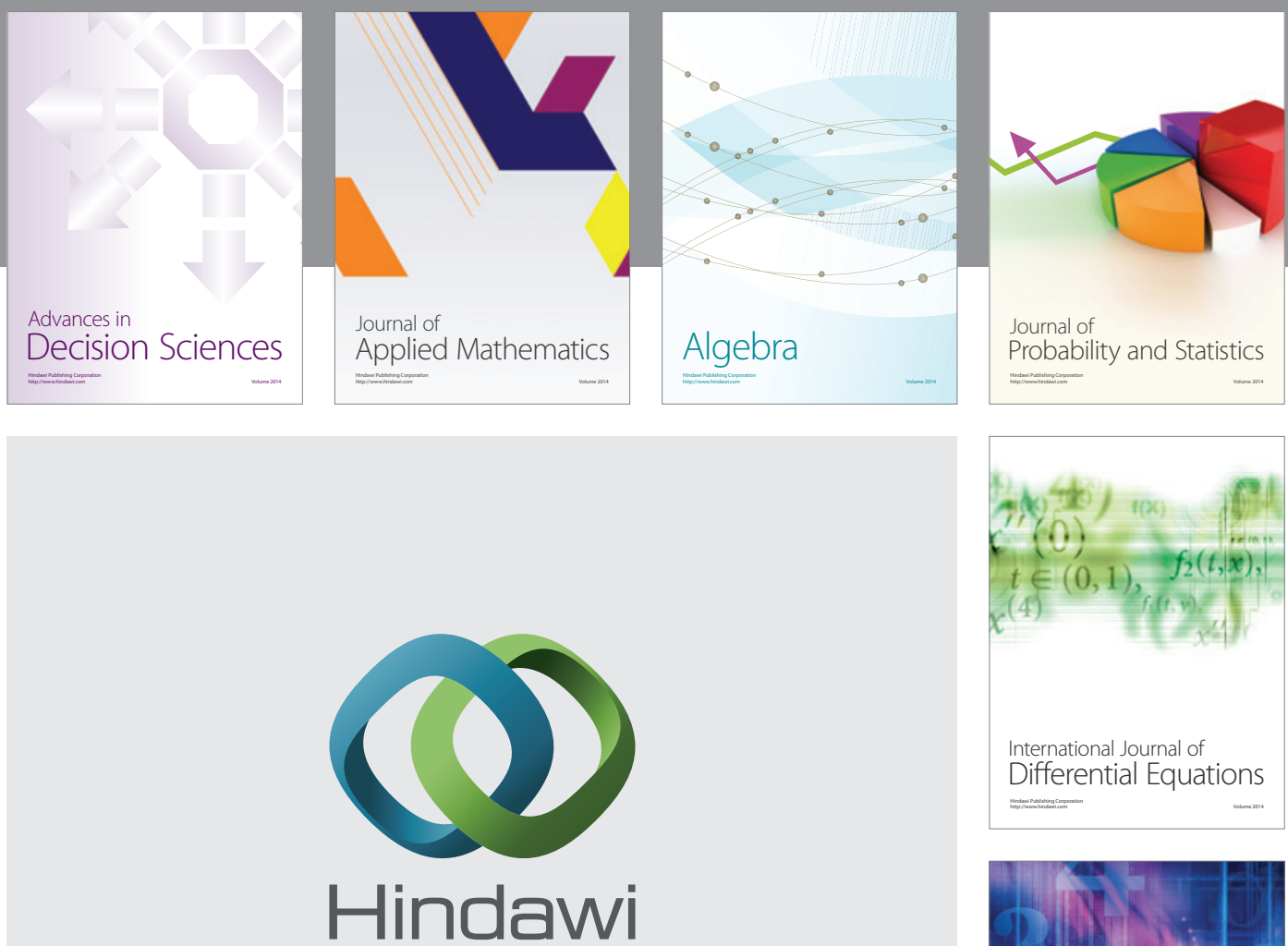

Submit your manuscripts at http://www.hindawi.com
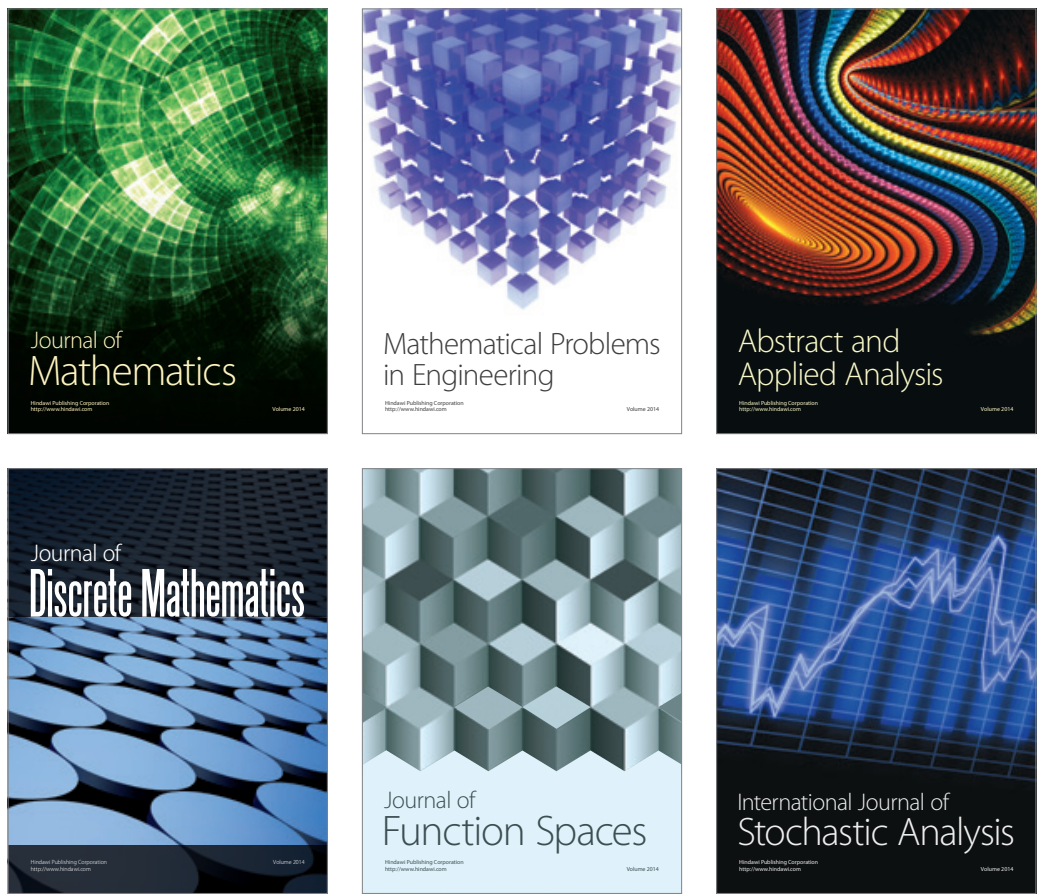

Journal of

Function Spaces

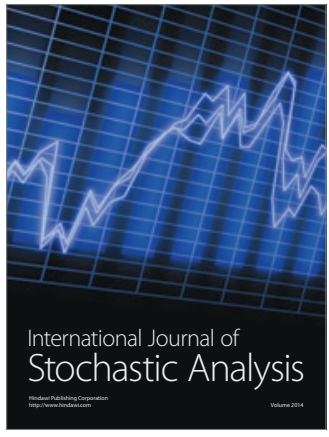

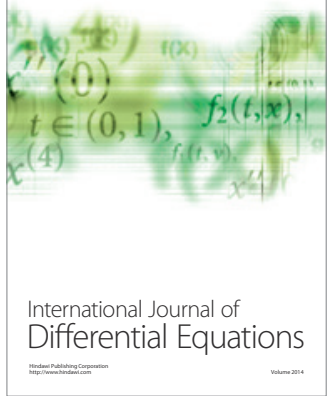
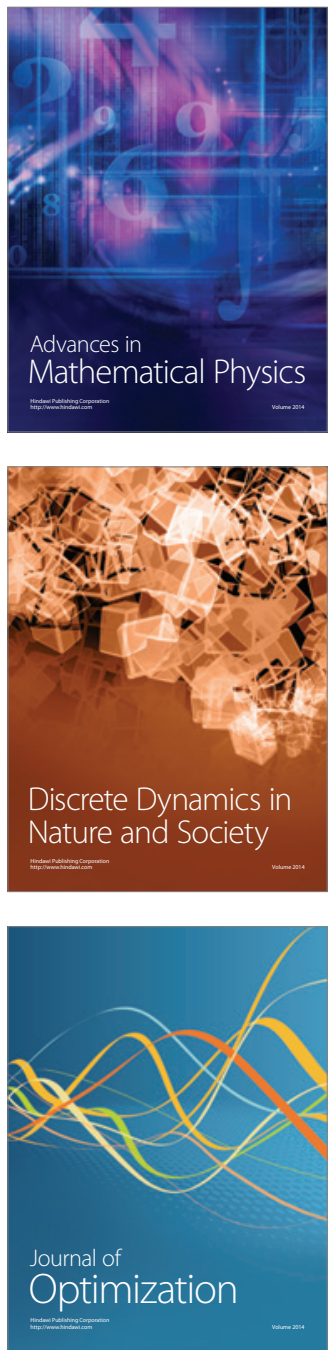